\title{
Avaliação Microbiológica e Físico-química em produtos industrializados à base de Tomate (Lycopersicon esculentum)
}

\section{Microbiological and Physical-chemical evaluation of industrialized tomato-based products (Lycopersicon esculentum)}

Thaynna Leocádio Trajano Lacerda Souza ${ }^{1 *}$, Gerlane Souza de Lima ${ }^{2}$, Indira Maria Estolano Macedo $^{1}$, Maria Karollyna Gomes da Silva ${ }^{1}$, Aline Gomes Santana ${ }^{1}$, Maria Lúcia Gurgel da Costa $^{2}$, Roberta Morgana da Mota Quirino ${ }^{2}$, Neide Kazue Sakugawa Shinohara ${ }^{1}$

\begin{abstract}
RESUMO
O tomate (Lycopersicon esculentum) é um fruto amplamente cultivado e consumido em todos os continentes, com propriedades nutricionais e antioxidantes importantes para a saúde humana. O objetivo do estudo foi promover a determinação físico-química e avaliação microbiológica em produtos industrializados de tomate. Foram realizadas análises de pH, Coliformes Totais, Escherichia coli, Salmonella sp. e Bolores e Leveduras em 59 amostras de produtos industrializados derivados do tomate (molhos, extratos, polpas e ketchups), adquiridas na região metropolitana do Recife (RMR). O pH apresentou valores seguros para crescimento de patógenos alimentares, o que pode ter contribuído para as contagens inferiores a $2 \log \mathrm{UFC}_{\mathrm{g}}{ }^{-1}$ de Coliformes Totais, E. coli, Salmonella sp. e parâmetros fúngicos. Estes resultados demonstram que estes produtos alimentícios se mostram satisfatórios para o consumo.
\end{abstract}

Palavras-chave: Qualidade; Segurança alimentar; pH; Microrganismos.

\begin{abstract}
The tomato (Lycopersicon esculentum) is a fruit widely cultivated and consumed on all continents, with nutritional and antioxidant properties that are important for human health. The aim of the study was to promote the physicochemical determination and microbiological evaluation of industrialized tomato products. Analyzes of $\mathrm{pH}$, Total Coliforms, Escherichia coli, Salmonella sp. and Molds and Yeasts were carried out in 59 samples of industrialized tomato-derived products (sauces, extracts, pulps and ketchups), acquired in the metropolitan region of Recife (RMR). The $\mathrm{pH}$ showed safe values for the growth of food pathogens, which may have contributed to counts below $2 \log \mathrm{UFC}^{-1}$ of Total Coliforms, E. coli, Salmonella sp. and fungal parameters. These results demonstrate that these food products are satisfactory for consumption.
\end{abstract}

Keywords: Quality; Food safety; pH; Microorganisms.

\footnotetext{
${ }^{1}$ Universidade Federal Rural de Pernambuco (UFRPE).

*E-mail: thaynna.leocadio0@gmail.com

2 Universidade Federal de Pernambuco (UFPE).
} 


\section{INTRODUÇÃO}

O tomate (Lycopersicon esculentum) é um dos frutos mais populares em todo o mundo (RATTANAVIPANON et al., 2021). Apesar da origem desconhecida, é considerado possivelmente nativo da América Latina, de onde foi exportado para a Europa a partir da Região Andina no século XVI (BERGOUGNOUX, 2014; LI, et al., 2020; VÉLEZ-TERREROS et al., 2021).

Atualmente, o tomate é uma das culturas agrícolas com maior impacto econômico, sendo cultivado e consumido em todo mundo, seja na forma in natura ou processado, como molhos, pastas, ketchups, sopas, sucos, concentrados e outros derivados (BERGOUGNOUX, 2014; VÉLEZ-TERREROS et al., 2021; RATTANAVIPANON et al., 2021; OLIVEIRA-BOUZAS et al., 2021). De acordo com a Organização das Nações Unidas para a Alimentação e a Agricultura (FAO), a produção mundial de tomate atingiu em 2019 aproximadamente 1.808 milhões de toneladas (VÉLEZ-TERREROS et al., 2021). Este vegetal apresenta alta perecibilidade por conter cerca de $90 \%$ de água quando cru, estando assim mais suscetível a possibilidade de multiplicação microbiana. A matéria seca é aproximadamente $50 \%$ constituída por carboidratos como frutose, glicose, e ainda 94\% de água livre, composição ainda passível de deterioração a longo prazo (OLVEIRABOUZAS et al., 2021).

O tomate representa uma importante fonte de ácido ascórbico (vitamina $\mathrm{C}$ ), potássio, ácido fólico, compostos fenólicos e carotenoides, como o licopeno (RAMÍREZ; KALLARACKAL, 2019; LI, et al., 2020; VÉLEZ-TERREROS, et al. 2021; RATTANAVIPANON, et al. 2021). Este último, é o carotenóide mais abundante no tomate (Fagundes et al., 2015; OLVEIRA-BOUZAS et al., 2021), devido à alta atividade antioxidante, possui propriedades anticancerígenas e protetora contra doenças cardiovasculares, aliado da dietética e que são bastante recomendados para consumo humano (BERGOUGNOUX, 2014).

O objetivo desse estudo foi o de promover a determinação de $\mathrm{pH}$ e avaliação microbiológica em produtos industrializados de tomate, por entender que o mercado consumidor desses produtos é crescente e permanente; logo uma comprovada sanidade e garantia das características de qualidade desses grupos de alimentos industrializados, propiciará o consumo seguro e consciente do consumidor.

\section{MATERIAL E MÉTODOS}


Foram analisadas 59 amostras de produtos industrializados derivados do tomate, adquiridas na região metropolitana do Recife (RMR), em embalagens de plástico rígido, tetra brik, vidros e sacos plásticos laminados. Divididos em 28 amostras de molho de tomate; 11 de extrato de tomate; 4 de polpa de tomate e 16 de ketchup. As amostras receberam codificação alfanumérica, como mostrado na Tabela 1.

Dentre os derivados de tomate analisados, muitos se diferenciam pela presença de diversas especiarias ou diminuição de algum ingrediente da fórmula original (light e diet). Nesse estudo, observou-se que os produtos estavam dentro do prazo de validade, cujo vencimento seria de no mínimo 90 dias.

Após a compra das mesmas, estas foram encaminhadas para realização dos ensaios físico-químico e microbiológico. Todas as amostras foram realizadas em duplicata para todos os ensaios realizados.

Tabela 1 - Codificação alfanumérica das empresas, produtos e categorias.

\begin{tabular}{|c|c|c|}
\hline Empresa & Quantidade de Produto & Designação \\
\hline $\mathbf{A}$ & 5 & $\begin{array}{l}\text { Molho (A1, A2) } \\
\text { Polpa (A3) } \\
\text { Ketchup (A4, A5) }\end{array}$ \\
\hline B & 4 & $\begin{array}{l}\text { Molho (B1, B2) } \\
\text { Ketchup (B3, B4) }\end{array}$ \\
\hline $\mathbf{C}$ & 4 & Molho $(\mathrm{C} 1, \mathrm{C} 2, \mathrm{C} 3, \mathrm{C} 4)$ \\
\hline D & 5 & $\begin{array}{l}\text { Molho (D1, D2, D3) } \\
\text { Polpa (D4) } \\
\text { Ketchup (D5) }\end{array}$ \\
\hline $\mathbf{E}$ & 3 & $\begin{array}{l}\text { Molho (E1, E2) } \\
\text { Extrato (E3) }\end{array}$ \\
\hline $\mathbf{F}$ & 4 & $\begin{array}{l}\text { Molho (F1) } \\
\text { Extrato (F2, F3) } \\
\text { Ketchup (F4) }\end{array}$ \\
\hline $\mathbf{G}$ & 3 & $\begin{array}{l}\text { Molho (G1) } \\
\text { Ketchup (G2, G3) }\end{array}$ \\
\hline $\mathbf{H}$ & 3 & $\begin{array}{l}\text { Molho (H1, H2) } \\
\text { Extrato (H3) }\end{array}$ \\
\hline I & 2 & Molho (I1, I2) \\
\hline $\mathbf{J}$ & 1 & Molho (J1) \\
\hline $\mathbf{K}$ & 1 & Ketchup (K1) \\
\hline $\mathbf{L}$ & 1 & Molho (L1) \\
\hline $\mathbf{M}$ & 1 & Molho (M1) \\
\hline $\mathbf{N}$ & 2 & $\begin{array}{l}\text { Molho (N1) } \\
\text { Extrato (N2) }\end{array}$ \\
\hline
\end{tabular}




\begin{tabular}{|l|c|l|}
\hline O & 1 & Molho (O1) \\
\hline $\mathbf{P}$ & 3 & $\begin{array}{l}\text { Molho (P1) } \\
\text { Extrato (P2) } \\
\text { Ketchup (P3) }\end{array}$ \\
\hline $\mathbf{Q}$ & 1 & Molho (Q1) \\
\hline $\mathbf{R}$ & 1 & Molho (R1) \\
\hline $\mathbf{S}$ & 2 & $\begin{array}{l}\text { Molho (S1) } \\
\text { Polpa (S2) }\end{array}$ \\
\hline $\mathbf{T}$ & 2 & $\begin{array}{l}\text { Extrato (T1) } \\
\text { Ketchup (T2) }\end{array}$ \\
\hline $\mathbf{U}$ & 2 & $\begin{array}{l}\text { Extrato (U1) } \\
\text { Ketchup (U2) }\end{array}$ \\
\hline $\mathbf{V}$ & 1 & Extrato (V1) \\
\hline $\mathbf{W}$ & 3 & Ketchup (W1, W2, W3) \\
\hline $\mathbf{Y}$ & 1 & Ketchup (Y1) \\
\hline $\mathbf{X}$ & 2 & $\begin{array}{l}\text { Extrato (X1) } \\
\text { Polpa (X2) }\end{array}$ \\
\hline $\mathbf{Z}$ & 1 & Extrato (Z1) \\
\hline & Fonte: autoria própria \\
\hline
\end{tabular}

\section{Determinação do pH}

Para determinação do pH, foi utilizado a sonda HACH HG 40D Multi, método de luminescência onde o eletrodo é colocado em contato direto com os produtos líquidos na análise laboratorial (IAL, 1982).

\section{Análise microbiológica}

As amostras foram coletadas em condições assépticas, pesadas e diluídas em meio de cultura para diluição/enriquecimento específico para cada microrganismo alvo. Foram utilizados kits comerciais da Compact Dry® (Nissui Pharmaceutical Co., Ltd., Tokyo, Japan) para contagem de Coliformes Totais, Escherichia coli, Salmonella sp. e Bolores e Leveduras (APHA, 2005; MACEDO et al., 2021). As análises foram realizadas em duplicata e os resultados foram expressos em UFC/g de amostra.

\section{RESULTADOS E DISCUSSÃO}

Nas Tabelas de 2 a 5 estão os resultados dos valores de $\mathrm{pH}$ para molhos de tomate, extrato de tomate, polpa de tomate e ketchup, respectivamente. Abaixo, na Tabela 2, observamos os resultados de $\mathrm{pH}$ obtidos nos ensaios para os molhos de tomate. Os 
resultados de $\mathrm{pH}$ das 28 amostras analisadas, variaram na faixa entre 4,13 a 4,85, caracterizando a alta acidez do produto comercial final.

Tabela 2 - Resultados de $\mathrm{pH}$ em molho de tomate de diferentes pontos da RMR.

\begin{tabular}{|c|c|}
\hline \multicolumn{2}{|c|}{ Molho de Tomate } \\
\hline Amostra & pH \\
\hline A1 & 4,22 \\
\hline A2 & 4,63 \\
\hline B1 & 4,17 \\
\hline B2 & 4,34 \\
\hline C1 & 4,42 \\
\hline $\mathrm{C2}$ & 4,49 \\
\hline C3 & 4,43 \\
\hline C4 & 4,55 \\
\hline D1 & 4,33 \\
\hline D2 & 4,54 \\
\hline D3 & 4,13 \\
\hline E1 & 4,45 \\
\hline E2 & 4,17 \\
\hline F1 & 4,21 \\
\hline G1 & 4,30 \\
\hline H1 & 4,36 \\
\hline H2 & 4,41 \\
\hline I1 & 4,31 \\
\hline $\mathbf{I 2}$ & 4,37 \\
\hline J1 & 4,39 \\
\hline L1 & 4,21 \\
\hline M1 & 4,45 \\
\hline N1 & 4,28 \\
\hline 01 & 4,31 \\
\hline P1 & 4,35 \\
\hline Q1 & 4,43 \\
\hline R1 & 4,85 \\
\hline S1 & 4,36 \\
\hline
\end{tabular}

Fonte: autoria própria

Os ácidos orgânicos presentes em alimentos influenciam o sabor, odor, cor, estabilidade e a manutenção de qualidade (CECCHI, 2003). A determinação de acidez pode fornecer um dado valioso na apreciação do estado de conservação de um produto alimentício. Um processo de decomposição, seja por hidrólise, oxidação ou fermentação, altera quase sempre a concentração dos íons de hidrogênio. Os métodos de determinação 
da acidez podem ser os que avaliam a acidez titulável ou fornecem a concentração de íons de hidrogênio livres, por meio do pH (IAL, 1985).

Já está bem estabelecido que a maioria dos microrganismos crescem melhor em valores de $\mathrm{pH}$ em torno de 7,0, e que alguns poucos crescem em $\mathrm{pH}$ abaixo de 4,0. As bactérias tendem a ser mais exigentes em termos de $\mathrm{pH}$ do que os bolores e leveduras, sendo as bactérias patogênicas ainda mais exigentes quando esse parâmetro intrínseco permanecer próximo a faixa de neutralidade (FORSYTHE, 2013; JAY, 2005). Portanto, o controle do $\mathrm{pH}$ a valores mais próximos a 4,0, garantem que microrganismos patogênicos e deteriorantes tenham maior dificuldade em se desenvolver, prática tecnológica muito empregada na produção industrial.

A acidez em molhos de tomate é de suma importância para avaliar e atestar sua qualidade, assim como o conhecimento do $\mathrm{pH}$ em produtos de tomate, pois dependendo dos resultados, microrganismos como o Bacillus coagulans, Clostridium botulinum e Clostridium butiricum, podem deteriorar ou servirem de veículo de contaminação cruzada, quando o produto for superior a pH 4,3 (EMBRAPA, 2003; SILVA et al., 2017).

$\mathrm{Na}$ Tabela 3, observamos os resultados de $\mathrm{pH}$ obtidos nos extratos de tomate. Os resultados de $\mathrm{pH}$ variaram na faixa de 4,12 a 4,47 nas 11 amostras analisadas.

Tabela 3 - Resultados de $\mathrm{pH}$ em extrato de tomate de diferentes pontos da RMR.

\begin{tabular}{|c|c|}
\hline \multicolumn{2}{|c|}{ Extrato de Tomate } \\
\hline Amostra & pH \\
\hline E3 & 4,32 \\
\hline F2 & 4,12 \\
\hline F3 & 4,35 \\
\hline H3 & 4,38 \\
\hline N2 & 4,26 \\
\hline P2 & 4,20 \\
\hline T1 & 4,39 \\
\hline U1 & 4,47 \\
\hline V1 & 4,24 \\
\hline X1 & 4,40 \\
\hline Z1 & 4,15 \\
\hline
\end{tabular}

Fonte: autoria própria

De acordo com Franco e Landgraf (2008) é desejável e seguro o pH inferior a 4,5 nos alimentos industrializados, para impedir a proliferação de microrganismos 
patogênicos no produto final, principalmente a contaminação pelo Clostridium botulinum, tão relacionado a graves surtos alimentares em alimentos enlatados, por sua característica anaeróbia e capacidade de multiplicação em substratos ácidos. Os resultados de $\mathrm{pH}$ apresentados na Tabela 3, informam que as amostras de extrato de tomate estavam dentro da faixa de $\mathrm{pH}$ segura do ponto de vista sanitário.

$\mathrm{Na}$ Tabela 4, observamos os resultados de $\mathrm{pH}$ medidos na polpa de tomate. Os resultados variaram de $\mathrm{pH}$ na faixa de 4,43 a 4,64 em 4 amostras analisadas.

Tabela 4 - Resultados de $\mathrm{pH}$ em polpa de tomate de diferentes pontos da RMR.

\begin{tabular}{|c|c|}
\hline \multicolumn{2}{|c|}{ Polpa de Tomate } \\
\hline Amostra & pH \\
\hline A3 & 4,46 \\
\hline D4 & 4,64 \\
\hline S2 & 4,43 \\
\hline X2 & 4,52 \\
\hline
\end{tabular}

Fonte: autoria própria

Segundo McGEE (2011), de todos os purês de hortaliças, o molho e o extrato de tomate são os mais conhecidos não só no ocidente como no restante do globo. Cerca de 2/3 dos sólidos do tomate são açúcares e ácidos orgânicos saborosos, e aproximadamente $20 \%$ são carboidratos das paredes celulares que têm algum poder de espessamento (10\% celulose, $20 \%$ pectina e $5 \%$ de hemicelulose). Diante dessas características sensoriais apreciáveis, faz-se jus a afirmativa da grande popularização dos derivados de tomate na culinária mundial.

$\mathrm{Na}$ Tabela 5, observamos os resultados de $\mathrm{pH}$ medidos nos ketchups. Os resultados variaram de $\mathrm{pH}$ na faixa de 3,59 a 4,26 em 16 amostras analisadas.

Tabela 5 - Resultados de $\mathrm{pH}$ em ketchup de diferentes pontos da RMR.

\begin{tabular}{|c|c|}
\hline \multicolumn{2}{|c|}{ Molho Ketchup } \\
\hline Amostra & pH \\
\hline A4 & 4,10 \\
\hline A5 & 4,16 \\
\hline B3 & 3,71 \\
\hline B4 & 3,71 \\
\hline D5 & 4,26 \\
\hline F4 & 3,96 \\
\hline
\end{tabular}




\begin{tabular}{|c|c|}
\hline G2 & 3,77 \\
\hline G3 & 3,85 \\
\hline K1 & 3,71 \\
\hline P3 & 3,68 \\
\hline T2 & 3,92 \\
\hline U2 & 3,80 \\
\hline W1 & 3,90 \\
\hline W2 & 3,59 \\
\hline W3 & 3,88 \\
\hline Y1 & 3,98 \\
\hline
\end{tabular}

Fonte: autoria própria

A quantidade de sal utilizada no ketchup e a acidez acética do produto final normalmente variam de 1,5 a $3,0 \%$ e 0,4 a $2,3 \%$, respectivamente. Os teores de sal e acidez são de grande importância para a segurança microbiológica do produto industrializado. Os ácidos geralmente utilizados são: vinagre como fonte de ácido acético, suco de limão e outros ácidos orgânicos, como o cítrico e o fosfórico. Do ponto de vista sensorial, acréscimos nas quantidades de ácidos geralmente requerem ajustes nas quantidades de açúcares, a fim de manter equilibrado o perfil sensorial do produto. Os açúcares presentes no ketchup são derivados da polpa de tomate e dos açúcares adicionados, que podem ser simplesmente sacarose ou misturas de sacarose, glicose em pó, xaropes de glicose ou frutose (BANNWART, 2006).

A qualidade do ketchup, principalmente no que se refere a cor, consistência, sabor e odor, depende da qualidade de cada ingrediente utilizado em sua formulação, especialmente da polpa de tomate. Os métodos de análise normalmente efetuados para avaliação e controle de qualidade de ketchup são os mesmos, ou similares, aos empregados para polpa de tomate (GOULD, 1992).

Com relação aos ensaios microbiológicos quanto à pesquisa de coliformes totais, Escherichia coli, Salmonella sp., do total de 59 (cinquenta e nove) amostras analisadas de produtos industrializados à base de tomate desta pesquisa, 100\% apresentaram contagem inferior a 2,0 $\log \mathrm{UFC} / \mathrm{g}^{-1}$. Esses resultados demonstram que as condições higiênicas sanitárias se mostram satisfatórias para consumo, pois confirmaram a ausência desses enteropatógenos alimentares (SILVA et al., 2017).

Entretanto, devemos observar esses resultados iniciais com cautela, pois o tomate e seus derivados são bastante susceptíveis a contaminação de origem fúngica (JAY, 2005), devido ao fato que esse grupo botânico apresenta um percentual médio de 94,1\% de água livre; $4 \%$ de carboidrato; $1 \%$ de proteína; $0,3 \%$ de gordura e 0,6 de cinzas 
(FENNEMA; DAMODARAN; PARKIN, 2018). Nessa pesquisa foram encontrados em 2 (duas) amostras de ketchup contagem fúngica na ordem de 1,0 $\log \mathrm{UFC} / \mathrm{g}^{-1}$ e 1,5 $\log$ $\mathrm{UFC} / \mathrm{g}^{-1}$. Nessas amostras, apesar de apresentar contagem de fungos, estas ainda se apresentam seguras para consumo do ponto de vista sanitário.

Ainda segundo Jay (2005) e Trabulsi e Alterthum (2015), o tomate pode ser atacado pela Drosophila melanogaster (mosca da fruta), que carrega em seu corpo esporos e fragmentos micelianos provenientes de frutas e vegetais deteriorados, causando fendas e rachaduras em frutas e vegetais saudáveis, propagando esporos fúngicos deteriorantes e patogênicos produtores de micotoxinas, podendo causar agravos graves na saúde da população consumidora.

\section{CONCLUSÃO}

De acordo com os resultados obtidos, podemos concluir que os derivados do tomate atenderam parâmetros higiênico-sanitários e a faixa de $\mathrm{pH}$ determinado por normas sanitárias para inibir o crescimento de enteropatógenos oportunistas. Além de ser um alimento funcional com propriedade antioxidante e antitumoral, sendo os derivados como os molhos, extratos, polpas e ketchup com maior concentração de licopeno, quando comparado com o tomate in natura.

\section{REFERÊNCIAS}

APHA. 2005. Standard Methods for the examination of water and wastewaters, 21 th edition, American Public Health Association, Washington.

BANNWART, G. C. M. C. Aplicação de Neotame em Catchup: Avaliação de Desempenho e Estimativa de Ingestão. Tese de Doutorado em Ciência dos Alimentos. Campinas - SP, cap. 1, p. 12-20, 2006.

BERGOUGNOUX, V. The history of tomato: from domestication to biopharming. Biotechnology advances, v. 32, n. 1, p. 170-189, 2014.

CECCHI, H. M. Fundamentos teóricos e práticos em análise de alimentos. 2 ed. revista - Campinas, SP - Editora da Unicamp. 2003

EMBRAPA. Cultivo de tomate para industrialização. 2006. Disponível em: < https://sistemasdeproducao.cnptia.embrapa.br/FontesHTML/Tomate/TomateIndustrial_ 2ed >. Acesso em: 16 de maio de 2021.

FENNEMA, O. R.; DAMODARAN, S.; PARKIN, K. L. Química de Alimentos de Fennema. 5 ed. Editora Artmed, 2018. 1120 p. 
FORSYTHE, S. J. Microbiologia da Segurança dos Alimentos. Porto Alegre: Artmed, 2013.

FRANCO, B. D. G. M.; LANDGRAF, M. Microbiologia dos alimentos. São Paulo: Atheneu, 2008.

GOULD, W. A. Tomato production, processing and technology. 3 ed., cap. 11, 1992.

IAL. INSTITUTO ADOLFO LUTZ. Normas Analíticas do Instituto Adolfo Lutz: Métodos químicos e físicos para análise de alimentos. 3. ed. São Paulo: IMESP, 1985.

JAY, J. M. Microbiologia dos alimentos. 6 ed. Porto Alegre, Artmed, 2005.

LI, N. et al. Tomato and lycopene and multiple health outcomes: Umbrella review. Food Chemistry, p. 128396, 2020.

MACEDO, I. M. E. et al. Influência do ultrassom na estabilidade microbiológica e físico-química de salsichas de saramunete (Pseudupeneus maculatus). Journal of Food Processing and Preservation, v. 1, p. e15580, 2021.

McGEE, H. Comida \& Cozinha: ciência e cultura da culinária. São Paulo: Martins Fontes, 2011.

OLVEIRA-BOUZAS, V. et al. Evaluation of a modified atmosphere packaging system in pallets to extend the shelf-life of the stored tomato at cooling temperature. Food Chemistry, v. 364, p. 130309, 2021.

RAMÍREZ, F.; KALLARACKAL, J. Tree tomato (Solanum betaceum Cav.) reproductive physiology: A review. Scientia horticulturae, v. 248, p. 206-215, 2019.

RATTANAVIPANON, W. et al. Effect of tomato, lycopene and related products on blood pressure: A systematic review and network meta-analysis. Phytomedicine, $p$. $153512,2021$.

SILVA, N. et al. Manual de métodos de análise microbiológica de alimentos e água, São Paulo: Blucher, 2017.

TRABULSI, L. R.; ALTERTHUM, F. Microbiologia. São Paulo: Atheneu, 2015.

VÉLEZ-TERREROS, P. Y. et al. Comparison of major nutrients and minerals between organic and conventional tomatoes. A review. Journal of Food Composition and Analysis, p. 103922, 2021.

Recebido em: 01/09/2021

Aprovado em: 20/09/2021

Publicado em: 27/09/2021 